\title{
Weathering promoted by biological activity enhances carbon storage inside large phyllosilicate particles in an acidic forest soil
}

\author{
DELPHINE DERRIEN ${ }^{1}$, INGRIDE KELLEN ${ }^{1}$, JAAFAR \\ GHANBAJA $^{2}$ AND MARIE-PIERRE TURPAULT ${ }^{1}$ \\ ${ }^{1}$ INRAE \\ ${ }^{2}$ Université de Lorraine, CNRS, IJL \\ Presenting Author: delphine.derrien@inrae.fr
}

Phyllosilicates have been proposed to dominantly contribute to SOM storage in neutral to basic soils through $\mathrm{Ca}^{2+}$ mediation. In acidic soils, their role in $\mathrm{C}$ storage has been largely overlooked in favor of the investigation of SOM interaction with metal oxides, while the low $\mathrm{pH}$ of the soil solution likely promotes their weathering and their reactivity towards organic moieties. For these reasons, we hypothesize that phyllosilicates also significantly contribute to $\mathrm{C}$ sequestration in acidic soil when subjected to weathering processes. We expect this contribution to peak during the initial weathering phase and in soil locations such as the forest floor, where the intense biological activity is known to alter minerals at the annual timescale.

To test this hypothesis, we performed a mineral mesh bag incubation of large vermiculite particles $(100-200 \mu \mathrm{m})$ in an acidic forest soil just below the forest floor. After 20 years the vermiculite particles were significantly weathered. Dissolution has released $10 \%$ of the structural elements, CEC has strongly decreased due to interlayer $\mathrm{Al}$ hydroxylation and secondary minerals have deposited on the outer- and inner-sheet surfaces. Fine-scale observations performed with SEM and TEM revealed that weathering has promoted the formation of nano- to microsized exfoliation spaces inside the vermiculite particles. Organic matter co-localized with calcium was entrapped in these exfoliation spaces, sometimes in association with Mn oxides or other secondary mineral deposits. Despite the acidic nature of the soil in the experimental site, the biological recycling of $\mathrm{Ca}$ oxalate and Ca-rich molecules releases unneglectable amounts of $\mathrm{Ca}$ in the soil solution in the forest floor. This $\mathrm{Ca}$ likely favors SOM interaction with the weathered surfaces of the exfoliation spaces, and may also act as a bridging agent between organic moieties.

These observations performed after only two decades in an acidic soil suggest that mineral weathering effectively favors $\mathrm{C}$ entrapment and protection inside phyllosilicate particles in hotspots of biological activity. Further investigations are needed to generalize this finding and to determine at which stage the progression of weathering will eventually induce the complete separation of vermiculite sheets and expose SOM to decomposers. 